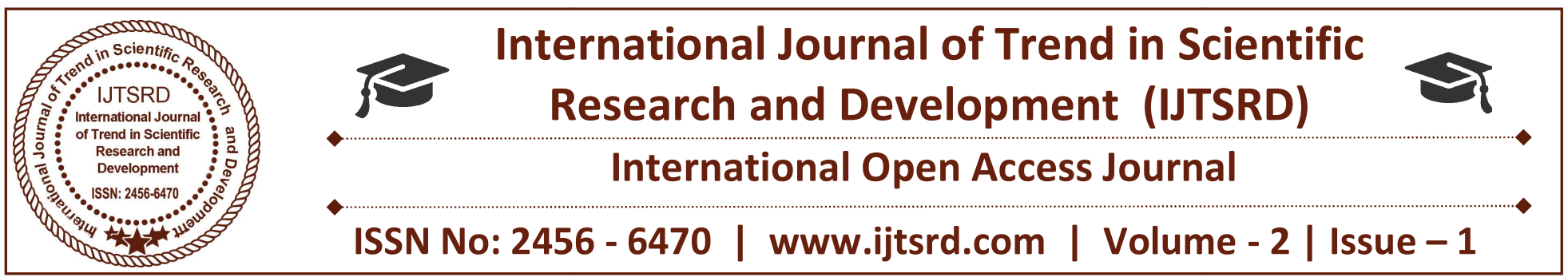

\title{
Deposition \& Characterization of Various Types of Coatings Development of Humidity Sensor
}

\author{
Aditya Vamshi Basa \\ A.V.College, Osmania \\ University, Hyderabad \\ Godisela Ram Murthy \\ Department of Applied Physics, National \\ Institute of Technology, Kurukshetra
}

\author{
K.Venu \\ Department of Geophysics, \\ Osmania University, Hyderabad \\ T.Aruna Kumari \\ Department of Physics, SRI SAI Degree \& P.G. \\ College, Osmania University, Hyderabad
}

\section{ABSTRACT}

The objective of the work was to study and learn about the thin film coatings design and development of various types of coatings. The study of various thin film coatings helped in the development of Humidity sensors. The development of these sensors depended basically on the change in resistance of TiO2. Films with respect to humidity

In the first chapter the description is about the background of thin films i.e. how the thin films were developed, their concepts and design of thin film coatings.

Feasibility studies for development of Humidity sensor and their characterization in third chapter. In the last chapter described about meteorology and applications of the thin films.

Keywords: film coatings, Humidity sensors

\section{INTRODUCTION}

Thin films are deposited on to bulk materials (any substrate) to achieve properties which are unattainable or not easily attainable in the substrates alone. Thin solid films of metals, dielectrics, semiconductors and combinations of these in the thickness range few angstroms $\left(1 \mathrm{~A}=10^{-10} \mathrm{~m}\right)$ to microns $\left(1 \mathrm{micron}=10^{-6} \mathrm{~m}\right)$ deposited on metallic and non-metallic substrates find enormous and diverse applications in science and technology.
In the present state of science and technology, thin films play an important role in the investigations of the fundamental properties of solids and in revolutionizing the basic concepts, quality and performance of science-based industrial products. In fact, they have crossed the frontiers of laboratory investigations and become the backbone of presentday electronic, optical and automotive industries as well as playing a pivotal role in the harnessing of solar energy.

In recent years, thin film science has grown worldwide into a major research area. The importance of coatings and the synthesis of new materials for industry have resulted in a tremendous increase of innovative thin film processing technologies. Currently, this development goes hand-in-hand with the explosion of scientific and technological breakthrough in microelectronics, optics and nanotechnology. These films are essential for a multitude of production areas, such as thermal barrier and wear protections, enhancing service life of tools and to protect materials against thermal and atmospheric influences. Here we concentrated only on optical thin films.

\section{METHODOLOGY}

1. Design of Thin film Coatings

$>$ Selection of method and materials

$>$ Spectral Characteristics 
$>$ Environmental conditions

$>$ Requirement / application

\section{Selection of Method}

Many types of deposition methods for thin film coating mainly those are

\section{1) Physical vapor deposition method}

1) Thermal evaporation

2) EB-gun

3) Sputtering

4) Ion deposition

2) Chemical vapor deposition methods

1) Electrode Deposition

2) Chemical vapor

3) Sol gel

4) Spray paralysis

\section{Selection of Material}

1. Substrate Material: Optical Glass (for optical coatings)

2. Substrate cleaning material: Iso propyl alcohol, teefal and acids $(\mathrm{HCl})$.

3. Substrate polishing material: Cerium Oxide

4. Coating materials

- Metals: Gold, Silver, Aluminum, Copper, Inconel, Platinum, Nickel.

- Dielectrics: Silicón dióxido (SiO2), Aluminum Tri oxide (A12O3)

- Conductors: Indium tin oxide (In2O3), Alloy of indium oxides etc.

- Semiconductors: Germanium, Silicon

- Fluorides: Magnesium fluoride etc

\section{EXPERIMENTAL PROCEDURE}

\section{Cleaning procedure of the substrate}

- Dip the substrate in the Dilute HCL, Then wash the substrate in ordinary water

- Clean the substrate with Teepol soap solution; again wash the substrate.

- Dip the substrate in distil water and Dry the substrate

- Finally clean the substrate in ultrasonic method using alcohol.

\section{Loading of substrate in coating plant}

First we must choose the suitable fixture for the substrate, because different size and shape of substrates used for different applications. Now fix the substrate in suitable fixture or zig and load the work holder. Masking is must for best uniformity of coating.

\section{Achieving of vacuum}

- Open the backing valve. The pressure should fall steadily to less than 0.1 torr as the diffusion pump is evacuated. Allow the cooling supply to flow.

- Switch on the diffusion pump, close backing valve and open roughing slowly. Select GH2 in pirani gauge.

- If pressure less than 0.1 torr. Close the roughing and open backing valve.

- Slowly open the baffle valve (high vacuum valve). As soon as the pressure starts rising in pirani GH1. And we may get high vaccum (i.e., $10^{-7}$ torr)

\section{High tension cleaning}

The static charge will affect the coating uniformity and adherence. In order to remove the static charge we use the High Tension cleaning. In the H.T cleaning the glow discharge removes any absorbed vapors and gases from the chamber internal surface.

- Close the high vacuum and open the baffle valve half. Switch on the H.T. button.

- Apply the low voltage from 0 to $160 \mathrm{~V}$ or high current about 3 amps and increase it slowly.

- Now apply the air in to the chamber through needle valve and observe the pressure in the chamber on gauge (0.1). Then rotate the substrate.

- Due to applied voltage to the aluminum foil it produce ions, bombards with the air molecules in the chamber takes place Glow discharge.

- After 5 to $7 \mathrm{~min}$, close air admittance valve and switch off the H.T cleaning button.

- On the completion of H.T cleaning, bring the variac to zero position.

- Close the air admittance valve and open baffle valve slowly keeping the backing pressure from rising above 0.2 torr.

\section{Apply L.T. (Low Tension) to the material}

- Switch on L.T. by selecting rotary switch, CB and L.T electrode selector switch.

- Rotate the evaporation source selector hand wheel until the required position is numbered. Its position also can be verified through the window.

- Slowly turn up the variac with the source shutter covered and observe the evaporation through the front window when evaporant is just melt. Uncover the source by means of shutter control knob, unless initial wetting of filament is needed 
in which case, leave the source covered until this is complete.

- Repeat the process with each filament if necessary, zero variac before changing position of the turret.

- When the evaporation is completed shutter the source, zero the variac switch off the L.T. (20Volts 170 Amps continuous, 200Amps intermittant) and C.B.

- Open the air admittance valve, raise the chamber selecting hoist up.

- Unload the work holder. Handle the fresh films with care.

\section{Shut down the unit}

- Evacuate the chamber down to $10^{-4}$, and then close the high vaccum valve.

- Switch off the pirani/penning and diffusion pump.

- Run the rotary pump for a period of 15-25 min keeping backing valve open. Close the backing valve and switch off rotary pump and close the cooling water supply.

- Switch off the main switch.

\section{Principle of glow discharge:}

- Glow discharge is used to remove the dust particles and atmosphere effects. When applied the (H.T) voltage to the anode and cathode, at chamber Low pressure up to $4 * 10^{\wedge}-4$ such as .Due to high voltage ionization will take place and, Highly positive ions moved to the cathode, and electrons (negative charge particles), moved to the anode .The below figure shows the diagram of glow discharge.

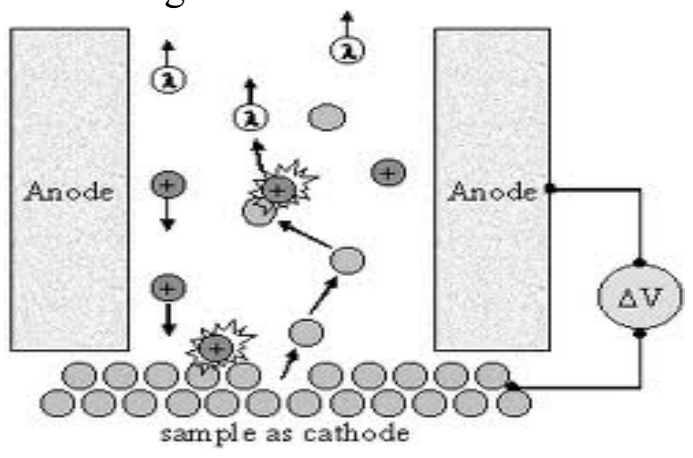

Fig 1

- Then when the velocity of positive ions is impinges or bombardment to the cathode, at the collision place, neutral ions will eject from the cathode. That ions remove the dust particles from the substrate. this is because of before going to the load the substrate we did rubbing, so that electro static force will create on the substrate so that the there is attraction force between the neutral atoms and substrate.

\section{THIN FILM COATING}

\section{HIGH REFLECTION COATING}

If we deposit a material of higher refractive index than that of glass substrate $(n=1.52)$, then we can increase the reflectance of bare substrate at certain wavelength depending up on optical thickness of the film.

The first condition is that the refractive index should be greater than that of incident medium and substrate

$$
\text { i.e. } \mathrm{n}_{0}<\mathrm{n}_{\mathrm{s}}<\mathrm{n}_{\mathrm{f}}
$$

Consider such a coating on a substrate as shown in figure 2.

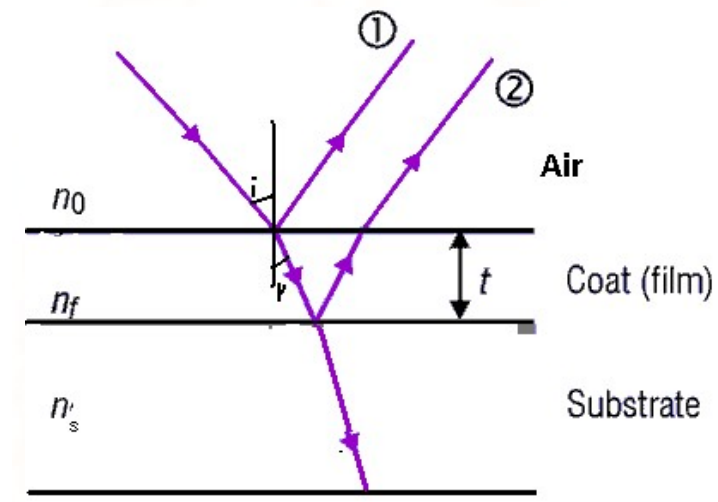

Figure 2

For maximum reflectance, the interference between the rays reflected from air-film and film-substrate interfaces should be constructive interference,

i.e. $\Delta=2 n_{f} t=(2 N+1) \lambda / 2$ (normal incidence) where $\mathrm{N}$ is an integer

$$
\text { For } \begin{aligned}
\mathrm{N} & =0, \\
t & =\frac{\lambda}{4 n_{f}}
\end{aligned}
$$

Optical thickness $=\mathrm{n}_{\mathrm{f}} \times t=\lambda / 4$ i.e. quarter wave thick.

By depositing a quarter wave thick $\mathrm{Al}$ on glass (1.52@550nm) we can increase reflectance from $80 \%$ (bare substrate) to $85-92 \%$.

\section{Fabrication of High Reflection Coating}

Material:

Aluminum in wire form

(99.9\% pure) 
Protection coating: Silicon monoxide $(\mathrm{SiO})$ in pallet form

Resistance source: helical filament and boat This coating has done in thermal plant ("HIND HIVAC" high vacuum coating plant). So generally every coating follows the same procedure but differ in resistance source. And before apply the low tension to $\mathrm{SiO}$, must enter the air into the chamber.

\section{Results}

Table 1

\begin{tabular}{|c|c|c|}
\hline S.No & $\begin{array}{c}\text { WAVELENGTH } \\
(\mathrm{nm})\end{array}$ & REFLECTANCE\% \\
\hline 1. & 400 & 93.9 \\
\hline 2. & 450 & 94.0 \\
\hline 3. & 500 & 94.2 \\
\hline 4. & 550 & 94.3 \\
\hline 5. & 600 & 94.2 \\
\hline 6. & 650 & 93.6 \\
\hline 7. & 700 & 92.9 \\
\hline
\end{tabular}

Lens Without Anti-Reflection Coating

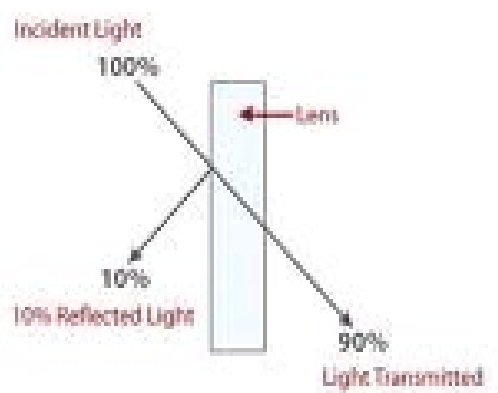

It depends for its operation on almost complete elimination of the light at the upper and lower of the two surfaces of the film n1.For complete elimination of two beams ,the reflected intensities at the upper and the lower boundaries of the film should be equal .which implies that the ratio of the refractive at each boundary should be equal;

$\mathrm{no} / \mathrm{n} 1=\mathrm{n} 1 / \mathrm{n} 2$;

$\mathrm{n} 1=V_{\mathrm{n} 0}{ }^{*} \mathrm{n} 2$.

This is the amplitude condition which shows that the film indexn1, should be intermediate between the indices of air and glass.

Anti-reflective or antireflection (AR) coatings are a type of optical coatings applied to the surface of

\section{ANTI REFLECTING COATING Single layer antireflection coating:}

When a beam $m$ of light traveling in air, refractive index, passes through the a glass plate, refractive index $\mathrm{n} 2$, a part of incident light will be reflected on the front an rear sufaces, as shown schematically.The reflectance $\mathrm{R}$ of a glass/air interface, defined as, the reflected intensity to the incident intensity, is a function of the difference in the refractive indices of the media and may be expressed for normal incident as:

$\mathrm{R}=($ no-n 1$) /(\mathrm{n} 0+\mathrm{n} 1) 2$

With ordinary glass plate $\mathrm{n} 2=1.52$ the original beam of light is decreased in intensity by this reflected amount ,approximately 4.1\%per surface ,making tota18.2\%.If approximate thin films, refractive index $\mathrm{n} 1$, are deposited on the surfaces, these reflection can be reduced, resulting in an effective improvement in the transmission of the glass. This process shown schematically in the below figure, is known as antireflection coating on the glass plate.

\section{Lens With Anti-Reflection Coating}

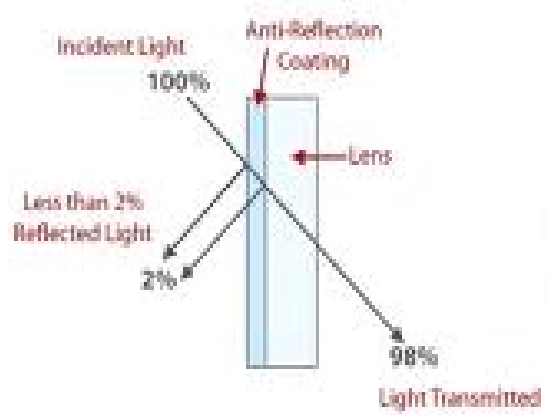

lenses or optical devices to reduce reflection. The glass elements in compound lens have a transmittance of only $92 \%$. The net transmittance of even a modest number of elements in series can therefore be quite low. Additionally, part of the light reflected at various surfaces eventually reaches the focal plane, where it appears as ghosts. Thus reducing the contrasts of images is especially important for zoom lenses used in photography, which would be completely unusable without AR coatings.

In other applications, the primary benefit is the elimination of the reflection itself, such as a coating on eyeglass lenses that makes the objects more visible. In complex systems such as a telescope, the 
reduction in reflections also improves the contrast of the image. This is specially important in astronomy.

Antireflection coatings can range from a simple single layer coating giving nearly zero reflectance to a multilayer system, having virtually zero reflectance over a range of wavelength.

\section{Principle:}

Consider a film of refractive index $n_{f}$ and thickness ' $t$ ' coated on a substrate of refractive index $n_{s}$ as shown in the figure 3 . When the light incident on such a film the light reflected from both interfaces interferes with each other constructively or destructively depending on the path/phase difference introduced between the two reflected rays.

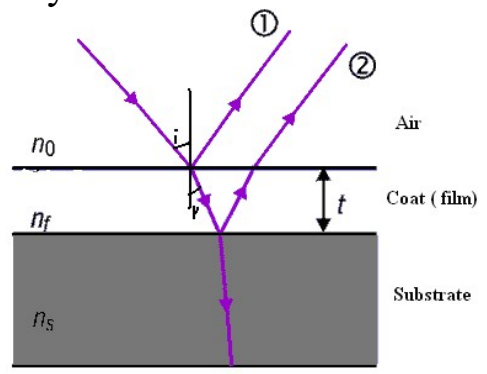

Path difference between rayl (reflected from air-film interface) and ray2 (reflected from film-substrate interface) is $\Delta=2 n_{f} t \cos r$

For normal incidence $\mathrm{i}=0^{0}$, therefore $\mathrm{r}=0^{0}$, therefore, $\Delta=2 n_{f} t$

For minimum reflectance the interference should be destructive

i.e. $\Delta=2 n_{f} t=(2 \mathrm{~N}+1) \lambda / 2 \quad$ where $\mathrm{N}$ is an integer

$$
\text { For } \mathrm{N}=0 \text {; }
$$

$$
t=\lambda / 4 n_{f}
$$

i.e. the thickness of coating should be quarter wave optical thickness.

Fresnel's equations for reflectivity at air-film and film-substrate interfaces are given by

$$
\begin{aligned}
& r_{1}=\frac{n_{0}-n_{f}}{n_{0}+n_{f}} \\
& r_{2}=\frac{n_{f}-n_{s}}{n_{f}+n_{s}}
\end{aligned}
$$

By considering multiple reflections at both interfaces we can get reflectance of the film

$$
\mathrm{R}=\frac{r_{1}{ }^{2}+r_{2}{ }^{2}+2 r_{1} r_{2} \cos 2 \Delta}{1+r_{1}{ }^{2} r_{2}{ }^{2}+2 r_{1} r_{2} \cos 2 \Delta}
$$

Where $\Delta$ is optical thickness of the film, which is equal to $\frac{\pi}{2}$ in this case

$\mathrm{R}=\frac{r_{1}^{2}+r_{2}^{2}-2 r_{1} r_{2}}{1+r_{1}^{2} r_{2}^{2}-2 r_{1} r_{2}}$

Using equations 1, 2 and 3 we can get reflectance in terms refractive indices as

$\mathrm{R}=\left(\frac{n_{0} n_{s}-n_{f}^{2}}{n_{o} n_{s}+n_{f}^{2}}\right)^{2}$

As we required zero reflected intensity, the reflectance $\mathrm{R}$ should be zero

$\mathrm{R}=\left(\frac{n_{0} n_{s}-n_{f}^{2}}{n_{o} n_{s}+n_{f}^{2}}\right)^{2}=0$

$$
n_{f}=\sqrt{n_{0} n_{s}}
$$

i.e. for single layer antireflection coating on a substrate, the refractive index of the film should be geometric mean of refractive indices of substrate and incident medium.

For antireflection coating on glass having refractive index 1.52 the film should have refractive index $n_{f}=\sqrt{1.52}=1.23$

However in nature no standard material has this refractive index. The material whose refractive index is closest to 1.23 is $\mathrm{MgF}_{2}(1.38 @ 550 \mathrm{~nm})$.other materials ,such as cryolite and chiolite, have lower indices ,but they are to some extent water soluble ,and films of these materials will not survive in humid environments.

\section{Double layer antireflection coating:}

The refractive indices of most glasses are too low for optimum efficiency of a single layer coating, If a more effective reduction in reflectance for various glass types required, this can be achieved with minimum of two films. multilayer $\lambda / 4$ whose indices decreases from the glass to the outer film surface .For zero reflection of the coating two $\lambda / 4$ layer systems ,air/n1/n2/glass, where $\mathrm{n} 1$ and $\mathrm{n} 2$ are the top and base layers respectively, should be in the following relation:

$$
\begin{aligned}
& \mathrm{n} 1 / \mathrm{n} 2=V_{\mathrm{ng}} \\
& \mathrm{n} 1=\mathrm{n} 2 \sqrt{ }_{\mathrm{ng}}
\end{aligned}
$$




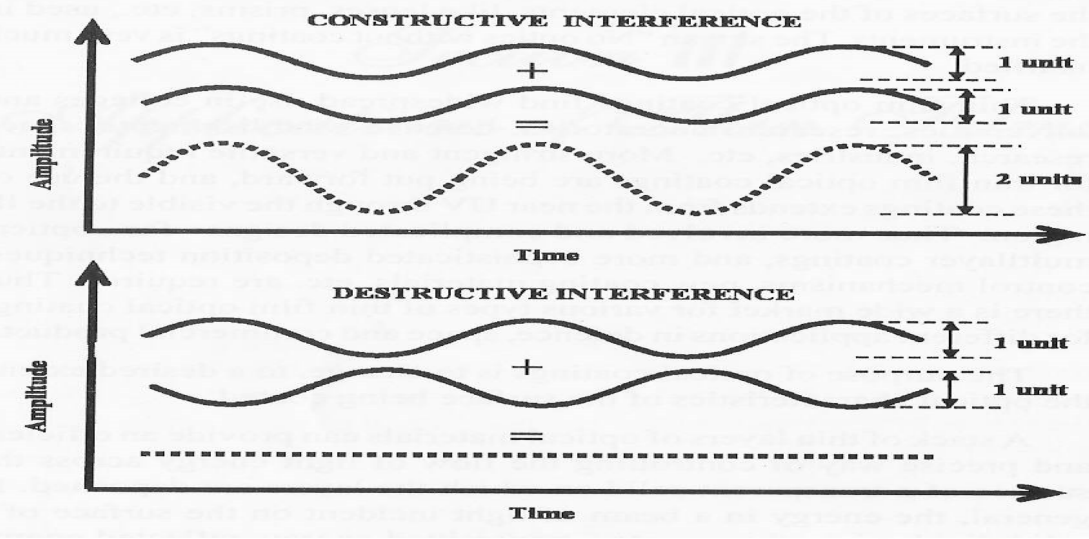

Figure 3

FABRICATION OF ANTIREFLECTION COATING

Materials: Zirconium oxide ( $\mathrm{ZrO} 2)$ in Tablet form Magnesium fluoride $\left(\mathrm{MgF}_{2}\right)$ Aluminum oxide (AL2O3) in pallet form

Resistance source: separate boats for each.

Procedure is same as for high reflection coating till the high tension cleaning is done.

- Then switch on the thickness monitor and adjust the transmittance to $92 \%$.
- Now apply low tension to the boat in order to melt the material for coating.

- Remove the shutter, read the thickness monitor simultaneously till desired quantity of transmittance is achieved. Nearly, we can achieve above $96 \%$ transmission.

- Turn off the low tension, Switch off the pump and leave the apparatus for 20-30 minutes till the substrate cools down.

- Then take out the coated sample carefully and measure its properties with the help of spectrophotometer.

\section{Results}

Table 2

\begin{tabular}{|l|l|l|l|l|}
\hline S.No & $\begin{array}{l}\text { WAVELENGTH } \\
(\mathrm{nm})\end{array}$ & $\begin{array}{l}\text { TRANSMITANCE } \\
(\%)\end{array}$ & $\begin{array}{l}\text { Single side anti } \\
\text { reflection } \\
\text { coating }\end{array}$ & $\begin{array}{l}\text { Both side } \\
\text { antireflection } \\
\text { coating }\end{array}$ \\
\hline 1. & 400 & 90.02 & 92.64 & 93.40 \\
\hline 2. & 450 & 91.24 & 93.51 & 95.29 \\
\hline 3. & 500 & 91.49 & 94.28 & 96.68 \\
\hline 4. & 550 & 91.56 & 94.54 & 97.10 \\
\hline 5. & 600 & 91.60 & 94.53 & 97.26 \\
\hline 6. & 650 & 91.73 & 94.46 & 97.22 \\
\hline 7. & 700 & 91.77 & 94.39 & 97.00 \\
\hline
\end{tabular}




\section{Results}

Table 3

\begin{tabular}{|c|c|c|}
\hline S.NO & $\begin{array}{l}\text { WAVE } \\
(\mathrm{nm})\end{array}$ & TRANSMISSION\% \\
\hline 1 & 400 & 2.30 \\
\hline 2 & 450 & 2.31 \\
\hline 3 & 500 & 2.36 \\
\hline 4 & 550 & 2.44 \\
\hline 5 & 600 & 2.56 \\
\hline 6 & 635 & 2.65 \\
\hline 7 & 650 & 2.68 \\
\hline 8 & 700 & 2.79 \\
\hline
\end{tabular}

\section{CONCLUSION:}

This Inconel coating has done on spectacles for laser applications. This inconel coating gives the neutral transmission of light over the selected wavelength range. In this way the intensity of light can be decreased uniformly to the desired value. The composition of Inconel is $9 \% \mathrm{Fe}, 76 \% \mathrm{Ni}$, and $15 \%$ $\mathrm{Cr}$ ) These type of coatings can be used for many other applications also like night driving filter etc.Material Inconel in wire form in Tungsten filament remaining procedure is samdescribed for other materials .

\section{REFERENCES}

1) Ronald R.Willey, a hand book of "Practical design and production of optical thin films".

2) H.A. Macleod, "Thin Film Optical Filters", $3^{\text {rd }}$ Edition, Institute of Physics Publishing.

3) James D. Rancourt, optical thin films users hand book.

4) High vacuum Technology: A Practical guide by M. H. Hablanian.

5) Practical guide of Hind High vacuum documents.

6) K.L. Chopra, a hand book of "Practical design and production of optical thin films".

7) Holland, "a hand book of optical thin film coatings".

8) Surface and Coatings Technology Volumes 120121, November 1999, Pages 303-312.

9) Vacuum engineering by N.S. HARRISH M.sc., M.Inst.p

10) www.google.com

11) www.wikipedia.com

12) www.freepatent.com

13) Langmuir 2007, 23, 3473-3477, Highly Sensitive Optical Detection of Humidity on Polymer/Metal Nanoparticle Hybrid Films 